\title{
Conformal invariance from scale invariance in nonlinear sigma models
}

\author{
Yu Nakayama \\ Department of Physics, Rikkyo University, Toshima, Tokyo 171-8501, Japan
}

(Received 10 July 2020; accepted 14 September 2020; published 25 September 2020)

\begin{abstract}
There exists a certain argument that in even dimensions, scale invariant quantum field theories are conformal invariant. We may try to extend the argument in $2 n+\epsilon$ dimensions, but the naive extension has a small loophole, which indeed shows an obstruction in nonlinear sigma models in $2+\epsilon$ dimensions. Even though it could have failed due to the loophole, we show that scale invariance does imply conformal invariance of nonlinear sigma models in $2+\epsilon$ dimension from the seminal work by Perelman on the Ricci flow.
\end{abstract}

DOI: $10.1103 /$ PhysRevD.102.065018

\section{INTRODUCTION}

The advent of conformal bootstrap approaches to critical phenomena (e.g., [1] for a review) raises a renewed interest in understanding about under which conditions the conformal symmetry emerges. Empirically, it is typically the case that scale invariance, Poincaré invariance (Euclidean invariance), and unitarity (reflection positivity) give rise to the enhanced conformal symmetry. Some arguments supporting this empirical fact exist in even space-time dimensions, in particular two [2] and four dimensions [3-6], but we do not have general arguments in odd dimensions, say, in three dimensions. ${ }^{1}$

In the perturbative regime, the (non)existence of scale invariant but not conformal field theory is closely related to the gradient nature of the renormalization group flow and the absence of the limit cycle [8-16]. Again, we do have supporting evidence for the gradient nature of the renormalization group flow in two and four dimensions. A crucial fact here is that the potential function for the gradient flow is given by Weyl anomaly coefficients at the conformal fixed point. They do exist in even dimensions but they do not exist in odd dimensions.

Without a general argument, it may be a natural idea to explore conformal invariance in odd dimensions by using the extrapolation of $d=2 n+\epsilon$ dimensions. Such approaches in various field theories are attempted in [2,17-19]. In this paper, we offer general discussions on how to obtain a

\footnotetext{
${ }^{1}$ Indeed, we do have an example of scale invariant but not conformal invariant field theories such as a free $U(1)$ gauge theory in three dimensions [7], so making the condition more precise is imperative.

Published by the American Physical Society under the terms of the Creative Commons Attribution 4.0 International license. Further distribution of this work must maintain attribution to the author(s) and the published article's title, journal citation, and DOI. Funded by SCOAP.
}

gradient flow of the renormalization group beta function in $d=2 n+\epsilon$ dimensions once we know that it is a gradient flow in $d=2 n$ dimensions. This typically implies conformal invariance in (perturbative) scale invariant fixed point in $d=$ $2 n+\epsilon$ dimensions if any.

We, however, find a small loophole in this argument, which indeed shows an obstruction in nonlinear sigma models in $d=2+\epsilon$ dimensions. The loophole is related to the question if the potential function for the gradient flow is bounded under the presence of the ambiguities in the beta functions. Even though the simple idea could have failed due to the loophole, we can still show that scale invariance does imply conformal invariance of nonlinear sigma models in $d=2+\epsilon$ dimension from the work by Perelman on the Ricci flow [20]. This, on the other hand, suggests that a general argument without a loophole would be quite nontrivial: at least it should directly imply Perelman's theorem on the Ricci flow.

\section{A SIMPLE ARGUMENT AND POSSIBLE LOOPHOLES}

We study a renormalization group flow of a local quantum field theory with Poincaré invariance. The properties of the renormalization group flow are characterized by the beta functions that appear in the trace of the energymomentum tensor.

Consider a general structure of the trace of the energymomentum tensor (in flat space-time):

$$
T_{\mu}^{\mu}=\beta^{I} O_{I}+s_{a} \partial_{\mu} J^{a}+\tau_{i} \square \Phi^{i}
$$

By using identities in a given field theory such as the nonconservation of the vector operator $\partial_{\mu} J_{a}^{\mu}=f_{a}^{I} O_{I}$, it is more convenient to rewrite the right-hand side as

$$
T_{\mu}^{\mu}=B^{I} O_{I} .
$$


We will use this scheme to evolve the coupling constant under the renormalization group flow: $\frac{d g^{I}}{d t}=B^{I}(g)$. Scale invariance demands $\beta^{I}=0$ while conformal invariance demands $B^{I}=0$. If $s_{a} \partial_{\mu} J_{a}^{\mu}$ is nonzero, it is sometimes called the Virial current. In most situations, one may remove $\tau_{i}$ by adding local counterterms to the action, but sometimes it gives a nontrivial consequence by adding further ambiguities in the definition of the beta functions.

In even dimensions $d=2 n$, there is a general argument that at the scale invariant fixed point all $B^{I}$ (rather than $\beta^{I}$ ) vanish, and the conformal invariance follows. One such argument is based on the gradient property of the beta functions. It claims that the beta functions are generated by a gradient flow:

$$
\frac{d g^{I}}{d t}=B^{I}=\chi^{I J} \frac{\partial a}{\partial g^{J}},
$$

with respect to a certain potential function $a(g)$, where we assume $\chi^{I J}(g)$ is positive definite. If this is the case, we can show

$$
\frac{d a}{d t}=B^{I} \frac{\partial a}{\partial g^{I}}=B^{I} \chi_{I J} B^{J} \geq 0,
$$

where $\chi_{I J}$ is an inverse of $\chi^{I J}$. In other words, $a(g(t))$ is monotonically decreasing along the renormalization group flow. ${ }^{2}$

In $d=2 n$ dimensions, $a(g)$ at the conformal fixed point is the Weyl anomaly coefficient which is positive definite. Therefore, if the theory under consideration can be deformed to be gapped, $a(g)$ cannot decrease forever. In the perturbative regime, we can argue that scale invariance demands $\frac{d a}{d t}$ is (at the worst) constant, but the constant must be zero since $a(g)$ is bounded. Then the positivity of $\chi_{I J}$ demands $B^{I}$ are all zero, implying that the scale invariant fixed points are actually conformal invariant.

In the literature, there have been substantial works on how to implement the above scenario in a concrete manner. We also realize that there are various subtle issues (e.g., if $\chi_{I J}$ can remain positive definite beyond the perturbation theories). We are not going to review such issues, but we refer [21] for a review.

In this paper, we simply assume the gradient flow nature of the beta functions in $d=2 n$ dimensions, and we would like to see if we can extend the above analysis in $d=$ $2 n+\epsilon$ dimensions. When we use the dimensional regularization with minimal subtraction, the beta functions $\tilde{B}^{I}$ in $d=2 n+\epsilon$ dimension and that of $d=2 n$ dimensions $B^{I}$ are related by

\footnotetext{
${ }^{2}$ Our convention is $t=\log \Lambda$ with cut-off $\Lambda$, and large $t$ corresponds to ultraviolet. Throughout the paper, we use the conventional term "monotonically decreasing" along the renormalization group flow, but it actually means monotonically increasing with respect to $t$.
}

$$
\tilde{B}^{I}=\epsilon k^{I}+B^{I},
$$

where $k^{I}$ may depend on the operator under consideration. ${ }^{3}$ We also note that this simple relation only holds in a particular renormalization scheme, and we will commit ourselves to such a scheme in the following.

Let us further assume we are working in the perturbative regime so that we may regard the field space metric as a unit matrix $\chi_{I J}=\delta_{I J}$. Then, if $B^{I}$ is a gradient flow, $\tilde{B}^{I}$ is a gradient flow as well:

$$
\tilde{B}^{I}=\chi^{I J} \frac{\partial \tilde{a}}{\partial g^{J}},
$$

where $\tilde{a}(g)=\frac{\epsilon}{2} k^{I} g^{I} g^{I}+a(g)$. Note that the gradient extension might fail beyond the perturbation theory in which $\chi_{I J}$ can be regarded as a constant. ${ }^{4}$

Now we can repeat the analysis in $d=2 n$ dimensions. If $\tilde{a}(g)$ were bounded, then we could argue $\tilde{B}^{I}=0$ at the scale invariant fixed point and then we would conclude that the fixed point is conformal invariant. Here is, however, a small loophole. In $d=2 n$ dimensions, $a(g)$ has a clear physical meaning such as the Weyl anomaly coefficient and it has a manifest positivity at the conformal fixed point. In $d=$ $2 n+\epsilon$ dimension, the precise physical meaning of $\tilde{a}(g)$ is unclear at this point and it could be unbounded.

Let us take a look at an example. In $d=4$ dimensions, the $\phi^{4}$ theory with the coupling constant $\lambda_{a b c d} \phi^{a} \phi^{b} \phi^{c} \phi^{d}$ has the beta function

$B_{a b c d}=\frac{1}{16 \pi^{2}}\left(\lambda_{a b e f} \lambda_{e f c d}+\lambda_{\text {acef }} \lambda_{e f b d}+\lambda_{a d e f} \lambda_{e f b c}\right)$,

so that in $d=4+\epsilon$ dimensions (being careful about the sign convention of $\epsilon$ ), we have

$$
\begin{aligned}
\tilde{B}_{a b c d}= & +\epsilon \lambda_{a b c d} \\
& +\frac{1}{16 \pi^{2}}\left(\lambda_{a b e f} \lambda_{e f c d}+\lambda_{a c e f} \lambda_{e f b d}+\lambda_{a d e f} \lambda_{e f b c}\right) .
\end{aligned}
$$

This is a gradient flow with respect to the potential

$$
\tilde{a}=+\frac{\epsilon}{2} \lambda_{a b c d} \lambda_{a b c d}+\frac{1}{16 \pi^{2}} \lambda_{a b c d} \lambda_{c d e f} \lambda_{e f a b} .
$$

We see that $\tilde{a}$ is monotonically decreasing along the (physical) renormalization group flow. We also see that at the scale invariant fixed point, we have $\tilde{B}^{a}=0$ with the enhanced conformal invariance. This is a favorable

\footnotetext{
${ }^{3}$ For example, if we consider Yukawa- $\phi^{4}$ theory in $d=4-\epsilon$ dimensions, the Yukawa coupling has $k=1 / 2$ while the scalar quartic coupling has $k=1$.

${ }^{4}$ By perturbation theory, we mean that we are close to a (conformal) fixed point. It does not necessarily mean that we are close to the Gaussian fixed point.
} 
situation in which the monotonicity of $\tilde{a}$ gives proof of conformal invariance.

\section{NONLINEAR SIGMA MODEL IN $d=2+\epsilon$ DIMENSIONS}

It is widely believed that the infrared renormalization group fixed point of the scalar $\phi^{4}$ theory in $d=4+\epsilon$ dimensions (with negative $\epsilon$ ) and the ultraviolet renormalization group fixed point of nonlinear sigma models in $d=2+\epsilon$ dimensions are in the same universality class if we extrapolate them to three dimensions. Since we have seen that the fixed points of $\phi^{4}$ theories are conformal invariant in the $d=4+\epsilon$ dimensions, we expect that the fixed points of the nonlinear sigma models in $d=2+\epsilon$ dimensions are also conformal invariant.

\section{A. A direct approach}

Let us consider the nonlinear sigma mode defined by the classical action

$$
S=\int d^{d} x G_{M N} \partial_{\mu} X^{M} \partial^{\mu} X^{N},
$$

whose target space $\mathcal{M}$ is a $D$-dimensional compact manifold with the metric $G_{M N}(X)$. In two dimensions, it is well known that the one-loop beta function is given by the Ricci tensor $R_{M N}(X)$ constructed out of $G_{M N}(X)$

$$
B_{M N}=\frac{d G_{M N}}{d t}=R_{M N},
$$

up to the ambiguity of the beta functions that can be added to the right-hand side [i.e., $D_{M} \partial_{N} \Phi(X)+D_{N} \partial_{M} \Phi(X)$ ] $[22,23]$. This ambiguity is associated with the dilaton coupling $\mathcal{R}^{(2)}(x) \Phi(X)$ or improvement of the energymomentum tensor. Here $\mathcal{R}^{(2)}(x)$ is the curvature of the $d$ dimensional "world-sheet". 5

In $2+\epsilon$ dimensions, the one-loop beta function becomes (again up to ambiguities)

$$
\tilde{B}_{M N}=-\epsilon G_{M N}+R_{M N},
$$

and the condition for scale invariance is

$$
\epsilon G_{M N}=R_{M N}+D_{M} V_{N}+D_{N} V_{M}
$$

for a particular vector field $V^{N}(X)$ on $\mathcal{M}$ with the covariant derivative $D_{M}$. Note that the term $D_{M} V_{N}+D_{N} V_{M}$ is the diffeomorphism induced by the vector field $V_{M}$ (i.e., Lie derivative of the metric), so the target space is "physically the same" with or without it. ${ }^{6}$

\footnotetext{
${ }^{5}$ We would like to avoid confusion with the target space Ricci scalar constructed out of $G_{I J}$.

${ }^{6} \mathrm{An}$ interesting application of this vector field can be found in [24].
}

If $V_{M}$ is a gradient vector: $V_{M}=\partial_{M} F(X)$ for a certain scalar function $F(X)$ on $\mathcal{M}$, then the scale invariant fixed point is conformal invariant because one can always remove it from the above ambiguity of the beta function. In [2], it was directly shown that $F=0$ when $\epsilon=0$ (even without using the ambiguity just mentioned). We would like to show a similar result when $\epsilon \neq 0$.

Acting $D_{M} D_{N}$ on (13) and using the Bianchi identity as well as (13) again, we obtain

$$
D^{M} D_{M} R+V^{M} D_{M} R=-2 R_{M N} R^{M N}+2 \epsilon R .
$$

Here $R=G^{M N} R_{M N}$ is the Ricci scalar. Let us pick a point $p$ such that $R$ takes the minimum value on $\mathcal{M}$. Since $D^{M} D_{M} R \geq 0$ and $D_{M} R=0$ at $p$, the left-hand side of (14) is non-negative. On the other hand, the right-hand side can be rewritten as

$$
\begin{aligned}
-2 R_{M N} R^{M N}+2 \epsilon R= & -2\left(R_{M N}-\frac{R}{D} g_{M N}\right)\left(R^{M N}-\frac{R}{D} g^{M N}\right) \\
& -2 R\left(\frac{R}{D}-\epsilon\right) .
\end{aligned}
$$

Here $R_{M N}-\frac{R}{D} g_{M N}$ is the traceless Ricci tensor. ${ }^{7}$ We will show that the right-hand side is nonpositive when $\epsilon \leq 0$.

Indeed, the trace of (13) says that $\int d^{D} x \sqrt{G} \frac{R}{D}=$ $\epsilon \int d^{D} x \sqrt{G}$, so $\epsilon$ is given by the mean curvature (divided by $D$ ). However, the minimum of the curvature is smaller than its mean, so $\frac{R(p)}{D} \leq \epsilon \leq 0$. Thus the right-hand side of (15) is a sum of two nonpositive terms, and they both must vanish. It means that $R=D \epsilon$ is a global constant, and $R_{M N}=\frac{R}{D} g_{M N}=\epsilon g_{M N}$, showing $V_{M}=0$. As we have promised $F=0$, and the scale invariant fixed point is conformal invariant. The target space is what is called the Einstein manifold.

This nice argument does not apply when $\epsilon>0$ and we cannot assign a definite sign on the right-hand side of (14). If this were literally true, we could conclude $V_{M}=0$ even without considering the possibility that it could be a gradient $V_{M}=\partial_{M} F$. On the contrary, it is known that when $\epsilon>0$ there does exist a solution of (13) with nontrivial $V_{M}=\partial_{M} F{ }^{8}$, and this approach must fail. We had to invent a more elaborate argument to show that scale invariance implies conformal invariance when $\epsilon>0$.

\section{B. A gradient approach 1}

Given success of Zamolodchikov's $c$-theorem in two dimensions [27], it is somewhat surprising that the explicit form of the monotonically decreasing $c$-function with the

\footnotetext{
${ }^{7}$ The idea that the traceless Ricci tensor is useful here is inspired by the work by Hamilton [25].

${ }^{8}$ The first compact one was discovered by Koiso [26]. We will also see a noncompact example later.
} 
gradient beta functions for the nonlinear sigma model was only available after the seminal work by Perelman [20] (see also related works [28-32]).

We consider the following $D$-dimensional target space action

$$
S[G, \phi]=\int d^{D} X \sqrt{G} e^{-2 \phi}\left(R+4 \partial_{M} \phi \partial^{M} \phi\right),
$$

and the $c$-function is defined by the minimum of $C[G]=$ $-\inf _{\phi} S[G, \phi]$ by varying $\phi$ that satisfies the normalization condition

$$
\int d^{D} X \sqrt{G} e^{-2 \phi}=1
$$

The target space action (16) is closely related to the effective action of the string theory. There $\phi$ is identified with a string dilaton and unconstrained, but here it is important to impose the normalization condition (17). To make it distinguished, it is sometimes called Perelman's dilaton or minimizer $\phi_{m}$.

This action can be used to derive the monotonic gradient flow of the beta function

$$
G_{I M} G_{J N} \frac{e^{2 \phi_{m}}}{\sqrt{G}} \frac{\delta C[G]}{\delta G_{I J}}=R_{M N}+D_{M} \partial_{N} \phi_{m}+D_{N} \partial_{M} \phi_{m},
$$

where Perelman's dilaton $\phi_{m}$ is not arbitrary but is fixed from $G_{M N}$ by minimizing $S[G, \phi]$. Remarkably this is identified with the beta function $B_{M N}$ of the metric, and in the particular scheme the renormalization group flow is generated by a gradient flow.

Let us now argue that scale invariance implies conformal invariance. In two dimensions, we see that at the scale invariant fixed point (18) must vanish to guarantee $\frac{d C[G]}{d t}=0$, and it directly implies the conformal invariance. Actually, repeating the argument in the previous subsection, we can further prove $\phi_{m}=$ const.

In $d=2+\epsilon$ dimensions, the beta function in the minimal subtraction scheme is given by

$$
B_{M N}=-\epsilon g_{M N}+R_{M N}+D_{M} \partial_{N} \Phi+D_{M} \partial_{N} \Phi .
$$

Here $\Phi(X)$ is an arbitrary scalar function on $\mathcal{M}$.

Now, as we discussed in Sec. II we may introduce the $c$-function in $d=2+\epsilon$ dimensions by

$$
\tilde{C}[G]=-2 \epsilon \int d^{D} x e^{-2 \phi_{m}} \sqrt{G}+C[G] .
$$

Here, in the first line, we do not vary $\phi$, which is already fixed in computing $C[G]$. This clearly gives a monotonically decreasing gradient flow in $2+\epsilon$ dimensions:

$$
\begin{aligned}
G_{I M} G_{J N} \frac{e^{2 \phi_{m}}}{\sqrt{G}} \frac{\delta \tilde{C}[G]}{\delta G_{I J}}= & -\epsilon G_{M N}+R_{M N}+D_{M} \partial_{N} \phi_{m} \\
& +D_{N} \partial_{M} \phi_{m},
\end{aligned}
$$

in a particular scheme where the ambiguity $\Phi$ in the beta function is fixed by Perelman's dilaton.

One may ask if this gives the proof that scale invariance implies conformal invariance in $2+\epsilon$ dimensions. The problem is that $\tilde{C}[G]$ is monotonically decreasing only for a particular $\phi_{m}$. We also do not know if $\tilde{C}[G]$ must be a constant at the scale invariant fixed point.

To see the difficulty in an example, let us consider the case with $G_{M N}=\delta_{M N}$. It is somewhat surprising but crucial to notice here that $B_{M N}$ is zero only if we supplement nontrivial $\Phi=\frac{\epsilon}{4} \delta_{M N} X^{M} X^{N}$. ${ }^{9}$ On the other hand, when we consider the flow from (21), the Perelman's dilaton $\phi_{m}$ is essentially derived in two dimensions so the obvious solution here is $\phi=$ const. This means that even if we have a scale invariant field theory, the $c$-function $\tilde{C}[G]$ is monotonically decreasing forever. ${ }^{10}$ This is nothing but the loophole we have mentioned in Sec. II.

\section{A gradient approach 2}

In the seminal paper [20], Perelman introduced the other monotonically decreasing functional, which he called the entropy. The direct renormalization group interpretation of Perelman's entropy in nonlinear sigma models in two dimension was not obvious, but we find that it has a direct connection with conformal invariance of nonlinear sigma models in $d=2+\epsilon$ dimensions. We will map the problem of finding a scale but not conformal fixed point in the nonlinear sigma model in $d=2+\epsilon$ dimension to the renormalization group flow in two-dimensions. Then we see that the stationarity of Perelman's entropy implies conformal invariance in $d=2+\epsilon$ dimensions for $\epsilon>0$.

Let us first map a scale invariant fixed point in $d=2+\epsilon$ dimensions to a nontrivial renormalization group flow in two dimensions. We will assume $\epsilon>0$. In $d=2+\epsilon$ dimensions, scale invariance implies that the metric satisfies

$$
\epsilon G_{M N}=R_{M N}+D_{M} V_{N}+D_{N} V_{M}
$$

for a certain vector field $V_{N}$. Let us now define the timedependent metric $G_{M N}(t)$ for $t>0$ by performing timedependent rescaling and time-dependent diffeomorphism on the time-independent metric $G_{M N}$ that satisfies (22): $G_{M N}(t)=\epsilon t \phi_{V}^{*}\left(G_{M N}(x)\right)$, where the pullback $\phi_{V}^{*}$ is induced by the diffeomorphism $\tilde{x}^{M}=x^{M}-\epsilon^{-1} \log (t) V^{M}$.

\footnotetext{
${ }^{9}$ In mathematics literature, it is known as the Gaussian Ricci soliton.

${ }^{10}$ Indeed it is given by $-e^{\frac{-D e t}{2}} V_{0}$ and the would-be fixed point is a singular metric of $G_{M N}=0$.
} 
Since the Ricci tensor is invariant under the rescaling of the metric [i.e., $R_{I J}(G)=R_{I J}(\alpha G)$ ], near $t=1$ the timedependent metric $G_{M N}(t)$ satisfies the Ricci-flow equation

$$
\frac{d G_{M N}(t)}{d t}=R_{M N}(t)
$$

where $R_{M N}(t)$ is the Ricci tensor for $G_{M N}(t)$. This time evolution is nothing but the renormalization group equation in two dimensions. In this way, we have mapped a scale invariant renormalization group fixed point in nonlinear sigma models $d=2+\epsilon$ dimensional to a particular renormalization group flow in two dimensions. ${ }^{11}$

We may now want to study the renormalization group flow of $G_{M N}(t)$ in the sense of the auxiliary two-dimensional nonlinear sigma model. We expect that it shows the monotonic behavior under the conventional $c$-function (or its generalization discussed in the previous section), but it is less useful in our setup because the metric typically blows up. At this point, Perelman introduced the other monotonically decreasing quantity, which he called the entropy. Consider the functional which explicitly depends on $t$ :

$$
\begin{aligned}
& S\left[t ; G_{M N}(t), \phi(t)\right] \\
& =-\int d^{D} X \sqrt{G(t)}\left(t\left(4 \partial_{M} \phi(t) \partial^{M} \phi(t)+R(t)\right)\right. \\
& \quad+2 \phi(t)-D)(4 \pi t)^{-\frac{D}{2}} e^{-2 \phi(t)} .
\end{aligned}
$$

The claim is that this functional is monotonically decreasing along the renormalization group flow. Note that Zamolodchikov's $c$-function does not depend on $t$ explicitly so it cannot be identified with the conventional $c$-function.

We study the time dependence of this functional under the generalized Ricci flow ${ }^{12}$

$$
\begin{aligned}
\frac{d G_{M N}(t)}{d t} & =R_{M N}(t) \\
\frac{d \phi(t)}{d t} & =\frac{1}{2} \square \phi-\partial_{M} \phi \partial^{M} \phi+\frac{R}{4}-\frac{D}{4 t} .
\end{aligned}
$$

The direct computation gives

\footnotetext{
${ }^{11}$ The discussion that follows does not explicitly use the fact that $\epsilon$ is small, but since we are neglecting the higher terms in the renormalization group beta functions, we effectively assume that $\epsilon$ is small.

${ }^{12}$ The time dependence is motivated as follows: we start with the gradient flow $\frac{d G_{M N}}{d t}=R_{M N}+D_{M} \partial_{N} \phi+D_{N} \partial_{M} \phi$ under the fixed measure $\sqrt{G}(4 \pi t)^{-\frac{D}{2}} e^{-2 \phi}$. The time dependence of $\frac{d \phi(t)}{d t}=$ $\frac{1}{2} \square \phi+\frac{R}{4}-\frac{D}{4 t}$ is induced from the time independence of the measure. Then we supplement the diffeomorphism of $V_{M}=$ $D_{M} \phi$ to make them the Ricci flow as in (25).
}

$$
\begin{aligned}
& \frac{d S\left[t ; G_{M N}(t), \phi(t)\right]}{d t} \\
& =\int d^{D} x \sqrt{G(t)} t\left(R_{M N}(t)+2 D_{M} D_{N} \phi(t)-\frac{1}{t} G_{M N}(t)\right)^{2} \\
& \quad \times(4 \pi t)^{-\frac{D}{2}} e^{-2 \phi} .
\end{aligned}
$$

Thus for $t>0, \quad S\left[t ; G_{M N}(t), \phi(t)\right]$ is monotonically decreasing along the renormalization group flow (i.e., monotonically increasing with respect to $t$ ). In particular, if $S\left[t ; G_{M N}(t), \phi(t)\right]$ is stationary, it satisfies

$$
R_{M N}(t)+2 D_{M} D_{N} \phi(t)-\frac{1}{t} G_{M N}(t)=0
$$

for any $\phi(t)$ that satisfies $(25) .^{13}$

We emphasize here that the fixed point of $S\left[t ; G_{M N}(t), \phi(t)\right]$ does not correspond to the renormalization group fixed point of two-dimensional nonlinear sigma models. Rather, it is related to a conformal invariant fixed point of nonlinear sigma models in $d=2+\epsilon$ dimensions as we will explain. Our strategy is that given the associated Ricci flow (23), we are going to find the specific $\phi(t)$ so that the entropy functional is stationary.

Let us now argue that scale invariant fixed point in $2+\epsilon$ dimension is conformal invariant from the monotonic properties of the entropy functional. For the purpose of finding a suitable $\phi(t)$ that makes our Ricci flow, which is induced from the scale invariant fixed point, stationary, we maximize $S\left[t, G_{M N}(t), \phi(t)\right]$ over $\phi(t)$ under the condition $\sqrt{G}(4 \pi t)^{-\frac{D}{2}} e^{-2 \phi}$ is fixed. The resulting $\bar{S}\left[t, G_{M N}(t)\right]=$ $\sup _{\phi} S\left[t, G_{M N}(t), \phi(t)\right]$ is also monotonically decreasing along the renormalization group flow. Now we note that $\bar{S}\left[t, G_{M N}(t)\right]$ is invariant under simultaneous scale change of $G_{M N}(t)$ and $t$ [i.e., $\left(G_{M N}, t\right) \rightarrow \alpha\left(G_{M N}, t\right)$ ]. We also note that $\bar{S}\left[t, G_{M N}(t)\right]$ is invariant under the diffeomorphism on $G_{M N}(t)$ thanks to the maximization over $\phi(t)$.

Due to these two properties of $\bar{S}\left[t, G_{M N}(t)\right]$, for the Ricci-flow solution induced from the scale invariant fixed point in $d=2+\epsilon$ dimensions, we find that $\bar{S}\left[t, G_{M N}(t)\right]$ is a constant near $t=1$ since the time evolution of $G_{M N}(t)$ is generated by the scale transformation and the diffeomorphism.

On the other hand, for generic Ricci flow, we know that the time dependence is given by (26). Therefore, when $\bar{S}\left[t, G_{M N}(t)\right]$ is stationary, it means

$$
R_{M N}(t)+2 D_{M} D_{N} \bar{\phi}(t)-\frac{1}{t} G_{M N}(t)=0
$$

\footnotetext{
${ }^{13}$ In order to assure the positivity, we have to assume that the target space has the Euclidean signature. This is related to unitarity; if the target space had the Lorentzian signature, unitarity of the nonlinear sigma model would be lost and we could not deduce (27).
} 
for a particular $\bar{\phi}(t)$ that maximizes the entropy functional. However, at $t=1$ the condition can be rewritten in terms of the original metric $G_{M N}$ as

$$
\epsilon G_{M N}=R_{M N}+2 D_{M} D_{N} F .
$$

This implies that the vector field $V_{M}=\partial_{M} F$ is a gradient and the scale invariant fixed point in $d+\epsilon$ dimension is conformal invariant.

As we have alluded above, unlike the case with $\epsilon<0$, we cannot conclude that $F$ is constant. Indeed, the manifold that satisfies the condition (29) is known as a gradient shrinking Ricci soliton (for positive $\epsilon$ ) and some nontrivial examples are available in the literature (see e.g., [26]). On the other hand, for negative $\epsilon$, it is known as a gradient expanding Ricci soliton, but we have already seen that they must be Einstein manifold and trivial (i.e., $F=0$ ).

\section{DISCUSSION}

We have shown that scale invariance implies conformal invariance in nonlinear sigma models in $d=2+\epsilon$ dimensions by using the mathematical result on the Ricci flow by Perelman. The monotonicity of Perelman's entropy along the renormalization group flow plays a crucial role, but it is not directly related to the renormalization group $c$-function in two dimensions because it explicitly depends on time. It is not the renormalization group $c$-function in $d=2+\epsilon$ dimensions either because it is only defined for scale invariant theories. It, however, indicates whether the fixed point in $d=2+\epsilon$ dimensions is conformal invariant or merely scale invariant.

It would be interesting to see if a similar function exists in other field theories than nonlinear sigma models at oneloop. In particular, Perelman's idea to map the scale invariant fixed point in $d=2+\epsilon$ dimension to the nontrivial renormalization group flow in two-dimension is not conventional in physics but may be of potential significance.

For the success of the mapping, it was crucial that the Ricci tensor is invariant under the rescaling of the metric. The similar thing may happen in one-loop gauge theories in $d=4+\epsilon$ dimensions. Suppose they are at the renormalization group fixed point

$$
0=-\epsilon g^{-2}+\beta_{0},
$$

where $\beta_{0}$ is a constant. We may now define the associated beta function in four dimensions from $g^{-2}(t)=\epsilon t g_{*}^{-2}$. It satisfies the $d=4$ dimensional renormalization group equation

$$
\frac{d g^{-2}(t)}{d t}=\beta_{0}
$$

at one-loop. Note that it was crucial that $\beta_{0}$ is a constant and does not depend on $g$.

Of course, at this point, we do not know if the analog of Perelman's entropy exists. Also, we admit that the mapping may not work at the higher loop order. Both in nonlinear sigma models and gauge theories, the two-loop term (e.g., $R_{M I J K} R_{N}^{I J K}$ ) is not invariant under the rescaling of the coupling constant, so the naive mapping does not work. It is therefore an interesting question to show conformal invariance of nonlinear sigma models in $d=2+\epsilon$ dimensions beyond the one-loop approximation.

\section{ACKNOWLEDGMENTS}

This work is in part supported by JSPS KAKENHI Grant No. 17K14301.
[1] D. Poland, S. Rychkov, and A. Vichi, Rev. Mod. Phys. 91, 015002 (2019).

[2] J. Polchinski, Nucl. Phys. B303, 226 (1988).

[3] M. A. Luty, J. Polchinski, and R. Rattazzi, J. High Energy Phys. 01 (2013) 152.

[4] A. Dymarsky, Z. Komargodski, A. Schwimmer, and S. Theisen, J. High Energy Phys. 10 (2015) 171.

[5] A. Dymarsky, K. Farnsworth, Z. Komargodski, M. A. Luty, and V. Prilepina, J. High Energy Phys. 02 (2016) 099.

[6] K. Yonekura, arXiv:1403.4939.

[7] S. El-Showk, Y. Nakayama, and S. Rychkov, Nucl. Phys. B848, 578 (2011).

[8] D. Wallace and R. Zia, Ann. Phys. (N.Y.) 92, 142 (1975).

[9] H. Osborn, Nucl. Phys. B363, 486 (1991).

[10] D. Friedan and A. Konechny, J. Phys. A 43, 215401 (2010).
[11] Y. Nakayama, Phys. Rev. D 87, 085005 (2013).

[12] J. F. Fortin, B. Grinstein, and A. Stergiou, J. High Energy Phys. 01 (2013) 184.

[13] B. Grinstein, A. Stergiou, and D. Stone, J. High Energy Phys. 11 (2013) 195.

[14] I. Jack and H. Osborn, Nucl. Phys. B883, 425 (2014).

[15] F. Baume, B. Keren-Zur, R. Rattazzi, and L. Vitale, J. High Energy Phys. 08 (2014) 152.

[16] I. Jack, D. Jones, and C. Poole, J. High Energy Phys. 09 (2015) 061.

[17] D. Dorigoni and V. S. Rychkov, arXiv:0910.1087.

[18] J. F. Fortin, B. Grinstein, and A. Stergiou, Phys. Lett. B 704, 74 (2011).

[19] S. Rychkov and A. Stergiou, SciPost Phys. 6, 008 (2019).

[20] G. Perelman, arXiv:math/0211159. 
[21] Y. Nakayama, Phys. Rep. 569, 1 (2015).

[22] D. Friedan, Phys. Rev. Lett. 45, 1057 (1980).

[23] D. H. Friedan, Ann. Phys. (N.Y.) 163, 318 (1985).

[24] D. Friedan, arXiv:1909.01374.

[25] R. S. Hamilton, Commun. Anal. Geom. 7, 695 (1999).

[26] N. Koiso, On rotationally symmetric Hamilton's equation for Kähler-Einstein metrics, in Recent Topics in Differential and Analytic Geometry, Adv. Stud. Pure Math. Vol. 18-I (Academic Press, Boston, MA, 1990), pp. 327-337.
[27] A. Zamolodchikov, JETP Lett. 43, 730 (1986).

[28] A. A. Tseytlin, Phys. Lett. B 194, 63 (1987).

[29] H. Osborn, Nucl. Phys. B308, 629 (1988).

[30] T. Oliynyk, V. Suneeta, and E. Woolgar, Phys. Lett. B 610, 115 (2005).

[31] T. Oliynyk, V. Suneeta, and E. Woolgar, Nucl. Phys. B739, 441 (2006).

[32] A. A. Tseytlin, Phys. Rev. D 75, 064024 (2007). 